\title{
Cancer associated fibroblasts are distinguishable from peri-tumor fibroblasts by biological characteristics in TSCC
}

\author{
PENGFEI BA ${ }^{1-3^{*}}$, XIAOJUAN ZHANG ${ }^{4 *}$, MIAO YU $^{5}$, LINXIA LI $^{6}$, XIAOYU DUAN ${ }^{7}$, MINGYING WANG $^{3}$, \\ SHUYAN LV ${ }^{3}$, GUO FU ${ }^{3}$, PISHAN YANG ${ }^{1,2}$, CHENGZHE YANG $^{8}$ and QINFENG SUN ${ }^{1,2}$ \\ ${ }^{1}$ Department of Periodontology, School of Stomatology, Shandong University; ${ }^{2}$ Shandong Provincial Key Laboratory of \\ Oral Tissue Regeneration, Shandong University, Jinan, Shandong 250012; ${ }^{3}$ Department of Periodontology, Weihai \\ Stomatological Hospital, Weihai, Shandong 264200; ${ }^{4}$ Department of Stomatology, Beijing Luhe Hospital Affiliated to \\ Capital Medical University, Beijing 101149; ${ }^{5}$ Department of Stomatology, Weifang People's Hospital, Weifang, \\ Shandong 261000; ${ }^{6}$ Department of Stomatology, Affiliated Hospital of Jining Medical University, Jining, Shandong 272000; \\ ${ }^{7}$ National Engineering Laboratory, WeGo Group Co., Ltd., Weihai, Shandong 264200; ${ }^{8}$ Department of Oral and \\ Maxillofacial Surgery, Qilu Hospital, Institute of Stomatology, Shandong University, Jinan, Shandong 250012, P.R. China
}

Received August 21, 2018; Accepted May 22, 2019

DOI: $10.3892 / \mathrm{ol} .2019 .10556$

\begin{abstract}
The aim of the present study was to investigate the differential biological characteristics between cancer-associated fibroblasts (CAFs) and peri-tumor fibroblasts (PTFs) in tongue squamous cell carcinoma (TSCC). The primary CAFs and PTFs from TSCC were obtained and purified. Cell morphology was observed, and the expression of $\alpha$-smooth muscle actin ( $\alpha$-SMA), vimentin and cytokeratin 19 (CK19) was detected by immunohistochemistry (IHC). The percentage of $\alpha$-SMA positive cells in CAFs and PTFs was calculated separately, and $\alpha$-SMA expression was further confirmed by western blot analysis. Cell viability and the expression of matrix metalloproteinase 2 (MMP2), stromal cell derived factorl (SDF-1) and transforming growth factor $\beta 1$ (TGF $\beta 1)$ in the purified fibroblasts was detected separately. CAFs and PTFs were attained and purified. Compared with PTFs, CAFs were long-fusiform shaped cells with reduced cytoplasm and variable size. CAFs crowded together in a disorderly manner when the cell density was increased, but this phenomenon did not occur with PTFs. IHC results verified that there was no significant difference between CAFs and PTFs in the percentage of
\end{abstract}

Correspondence to: Dr Qinfeng Sun, Department of Periodontology, School of Stomatology, Shandong University, 44-1 Wenhuaxi Road, Jinan, Shandong 250012, P.R. China

E-mail: sunqinfeng@sdu.edu.cn

Dr Chengzhe Yang, Department of Oral and Maxillofacial Surgery, Qilu Hospital, and Institute of Stomatology, Shandong University, 107 West Wen Hua Road, Jinan, Shandong 250012, P.R. China E-mail: yangchengzhe19@163.com

${ }^{*}$ Contributed equally

Key words: tongue squamous cell carcinoma, tumor microenvironment, cancer-associated fibroblasts, peri-tumor fibroblasts, cell phenotype cells staining positive for $\mathrm{CK} 19$ and vimentin $(\mathrm{P}>0.05)$; the percentage of positive staining cells for $\alpha$-SMA in CAFs was significantly higher compared with that in PTFs $(\mathrm{P}<0.001)$ Western blot analysis showed that $\alpha$-SMA expression in CAFs was 4.3-fold higher compared with that in PTFs $(\mathrm{P}<0.001)$. A Cell Counting Kit- 8 assay indicated that the viability of CAFs increased significantly compared with that in the PTFs $(\mathrm{P}<0.05)$. Reverse transcription-quantitative polymerase chain reaction and ELISA analysis showed that the expression of MMP2, SDF-1 and TGF $\beta 1$ in CAFs was higher compared with that in PTFs $(\mathrm{P}<0.05)$. CAFs are distinguishable from PTFs with respect to their morphology, cellular phenotype, cell viability and pro-carcinogenic cytokine expression.

\section{Introduction}

Tongue squamous cell carcinoma (TSCC) is one of the most common oral cancer types, usually characterized by the early occurrence of lymph node metastasis and poor prognosis (1-4). Though the precise mechanism of TSCC tumorigenesis remains unclear, it has become clear that the initiation and progression of tumors depends not only on the epithelial cells, but also on the interactions between the tumor stroma and tumor cells $(5,6)$. Cancer associated fibroblasts (CAFs), also named 'activated fibroblasts', are the most abundant stromal cell types of the tumor stroma $(7,8)$. CAFs modulate tumor angiogenesis, remodeling of the extracellular matrix and epithelial-to-mesenchymal transition, and also contribute to tumor recurrence and drug resistance $(9,10)$, and thus play a major role in the initiation and progression of carcinomas $(6,11)$. Therefore, CAFs would be effective targets for cancer treatment; the present study on CAFs may be beneficial for investigating the mechanisms of tumorigenesis and tumor progression.

CAFs have been identified to differ phenotypically and functionally from normal fibroblasts (NFs) $(12,13)$. Compared with fibroblasts from normal oral mucosal tissue, CAFs are 
characterized by higher expression of $\alpha$-SMA and a distinct morphology (13). This is why NFs are often used as the control when CAFs are studied (14). However, whether the behavior, phenotype and function of peri-tumor fibroblasts (PTFs) are different from CAFs in TSCC is not clear. In the present study, CAFs and PTFs were cultured from specimens of TSCC and peri-tumor tissues (confirmed by histology), from which fibroblasts were purified, and subsequently their differential biological characteristics by means of morphology observation, cell surface marker $[\alpha$-smooth muscle actin ( $\alpha$-SMA), vimentin and cytokeratin 19 (CK19)] expression, cell viability and pro-carcinogenic cytokine expression was investigated. This information, on the one hand, will provide some benefits in further understanding stromal cell changes in tumor development, and, in turn, understanding the mesenchymal-epithelium interactions in carcinogenesis. On the other hand, it may provide further experimental evidence for PTFs as a control in the examination of CAFs in TSCC.

\section{Materials and methods}

Tissue collection. TSCC specimens and 'histologically normal' peri-tumor tissues (at least $1 \mathrm{~cm}$ from the outer tumor margin) were collected from primary tumors from 6 patients (2 males and 4 females, age 40-65 years) who underwent surgical resection at the Qilu Hospital of Shandong University between March 2016 and January 2017. The inclusion criteria included: Age 18-75 years; a Karnofsky score (15) $\geq 60 \%$, and a life expectancy $\geq 3$ months; white blood cell $\geq 4.0 \times 10^{9} / 1$, absolute neutrophil count $\geq 2.0 \times 10^{9} / 1$, platelet count $\geq 100 \times 10^{9} / 1$, and hemoglobin $\geq 100 \mathrm{~g} / \mathrm{l}$; alanine transaminase and aspartate transaminase $<2.5 \mathrm{x}$ the upper limit of normal, total bilirubin and serum creatinine $<1.5 x$ the upper limit of normal. Patients were excluded if they had distant metastasis or other types of cancer, had undergone surgery involving primary tumor or lymph nodes (except diagnostic biopsy), had received prior radiotherapy or chemotherapy, presented with other malignancies within 5 years, or had creatinine clearance $<30 \mathrm{ml} / \mathrm{min}$. All the resections (TSCC and peri-tumor tissues) were confirmed by clinical and histopathological examination, according to the World Health Organization and the 2010 criteria of the International Union Against Cancer (16), All research procedures were approved by the Medical Ethics Committee of Qilu Hospital, Shandong University (2016015) and informed consent was obtained from all the participants.

Separation, cultivation and purification of CAFs and PTFs. The obtained tissues (tumor or peri-tumor) were immersed in Dulbecco's modified Eagle's medium (DMEM; HyClone; GE Healthcare Life Sciences) supplemented with $2 \%$ penicillin-streptomycin (HyClone; GE Healthcare Life Sciences) for at least $30 \mathrm{~min}$, and then washed with PBS (HyClone; GE Healthcare Life Sciences) three times. The tissues were minced with scalpels into $1.0 \times 1.0 \times 1.0 \mathrm{~mm}^{3}$ fragments and tiled on the bottom of a culture bottle. The cells were cultured in DMEM supplemented 20\% FBS (HyClone; GE Healthcare Life Sciences) and $1 \%$ penicillin-streptomycin. After $3 \mathrm{~h}$, the culture bottle was turned over. Cultures were incubated at $37^{\circ} \mathrm{C}$ in a humidified atmosphere of $5 \% \mathrm{CO}_{2}$ and cell growth was observed under an inverted microscope (magnification, x100).
Upon reaching $90 \%$ confluence, the areas which epithelioid cells had grown in clusters were marked on the culture bottle when the cells were observed under the phase-contrast microscope (magnification, x100), and according to the markers, epithelioid cells were wiped with a cotton stick dipped in $10 \mu \mathrm{l}$ of trypsin. The remaining cells were digested with $0.25 \%$ trypsinase for $1 \mathrm{~min}$; digestion was terminated with $3 \mathrm{ml}$ of medium containing serum, and cells were sub-cultured into fresh bottles. Cells at passage (P)3-P5 were used in subsequent experiments.

Cell morphology observation. Morphology of the purified P3-P5 CAFs and PTFs was observed using a phase-contrast microscope.

Immunohistochemistry (IHC). Coverslips with attached fibroblasts (at a density of $3 \times 10^{4}$ cells $/ \mathrm{cm}^{2}$ ) were fixed with $4 \%$ paraformaldehyde for $10-20 \mathrm{~min}$ at room temperature. IHC procedures using the SP kit (cat. no. SP9002; OriGene Technologies, Inc.) were performed according to the manufacturer's instructions. The fixed cells were washed in PBS three times, permeabilized with $0.05 \%$ Triton for $5 \mathrm{~min}$, and blocked with $4 \%$ bovine serum albumin (cat. no. A8010; Beijing Solarbio Science $\&$ Technology Co., Ltd.) for $30 \mathrm{~min}$ at $37^{\circ} \mathrm{C}$. Subsequently, the coverslips were incubated at $4^{\circ} \mathrm{C}$ in a moist chamber overnight with the following primary mouse anti-human monoclonal antibodies: Anti-human CK19 (dilution, 1:100; cat. no. BM3267), anti-human vimentin (dilution, 1:100; cat. no. BM0135), and anti-human $\alpha$-SMA (dilution, 1:100; cat. no. BM0002; all Wuhan Boster Biological Technology, Ltd.). PBS was used as the negative control, in place of the primary antibody. After incubation with HRP-conjugated rabbit-anti-mouse secondary antibody (dilution, 1:100 cat. no. BA1048; Wuhan Boster Biological Technology, Ltd.) for $30 \mathrm{~min}$ at room temperature, the coverslips were counterstained with Mayer hematoxylin (cat. no. G1080; Beijing Solarbio Science \& Technology Co., Ltd.) and observed under a light microscope (magnification, x400; Olympus BX53; Olympus Corporation, Tokyo, Japan,). Cells showing light-brown or yellow-brown grains in the cytoplasm were classified as positively stained.

Western blot analysis. CAFs/PTFs were seeded into 6-well plates $\left(1 \times 10^{5}\right.$ cells/well) overnight. Following this, whole-cell proteins were extracted using RIPA buffer (cat. no. R0020; Beijing SolarbioScience \& TechnologyCo.,Ltd.) andquantitated using a bicinchoninic acid protein assay kit (cat. no. PC0020; Beijing Solarbio Science \& Technology Co., Ltd.), according to the manufacturer's protocols; the proteins (10 $\mu \mathrm{g} /$ lane $)$ from different samples were separated via 10\% SDS-PAGE (cat. no. AR0138; Wuhan Boster Biological Technology, Ltd.) and transferred to polyvinylidene fluoride (PVDF) membranes (cat. no. ISEQ00010; EMD Millipore). The PVDF membranes were blocked with blocking buffer containing 5\% skimmed milk for $1 \mathrm{~h}$ at room temperature before incubation with the primary $\alpha$-SMA antibody (dilution, 1:200; cat. no. BM0002; Wuhan Boster Biological Technology, Ltd.) overnight at $4^{\circ} \mathrm{C}$. Protein bands were detected using the appropriate secondary antibodies (dilution, 1:2,000; cat. no. BA1092; Wuhan Boster Biological Engineering Co., Wuhan, China) for $1 \mathrm{~h}$ at $37^{\circ} \mathrm{C}$. and ECL reagents (cat. no. 17-373SP; EMD Millipore; Merck 


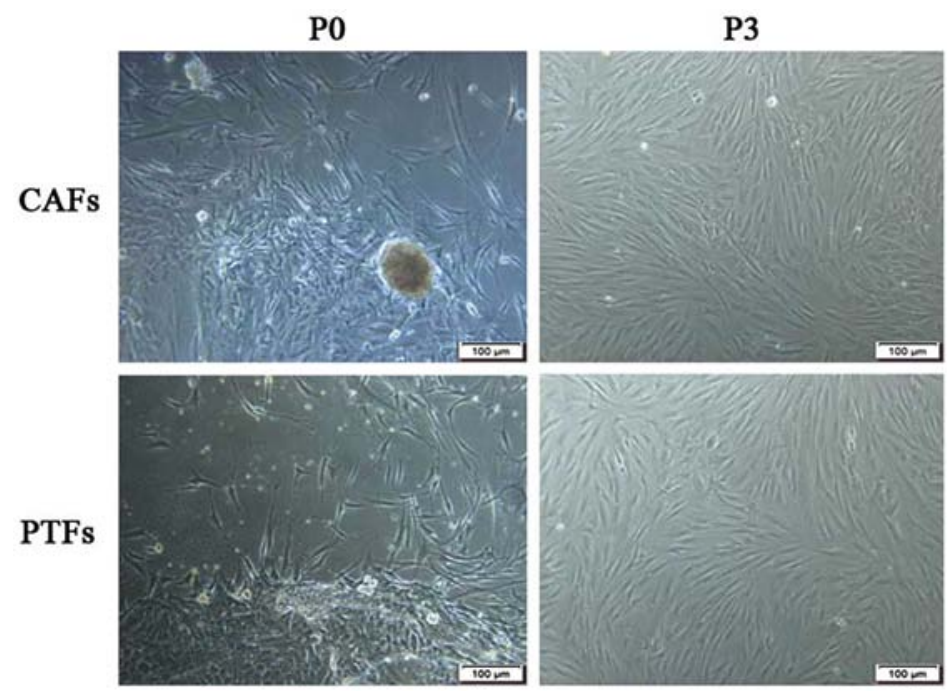

Figure 1. Separation, cultivation and purification of tongue CAFs and PTFs. The fibroblasts and epidermoid cells grew from the edge of the tissue at P0; no epithelioid cells or tumor cells were mixed at P3 after purification. CAFs, cancer-associated fibroblasts; PTFs, peri-tumor fibroblasts; P, passage.

A

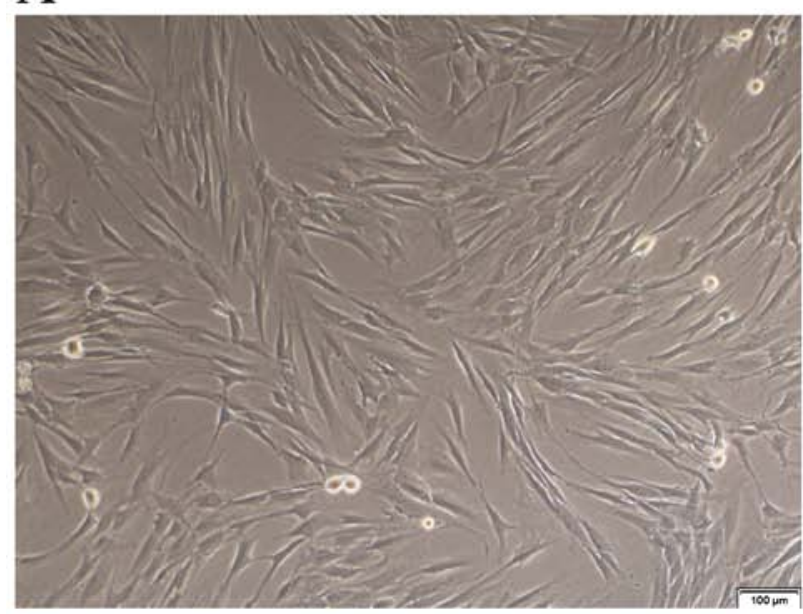

B

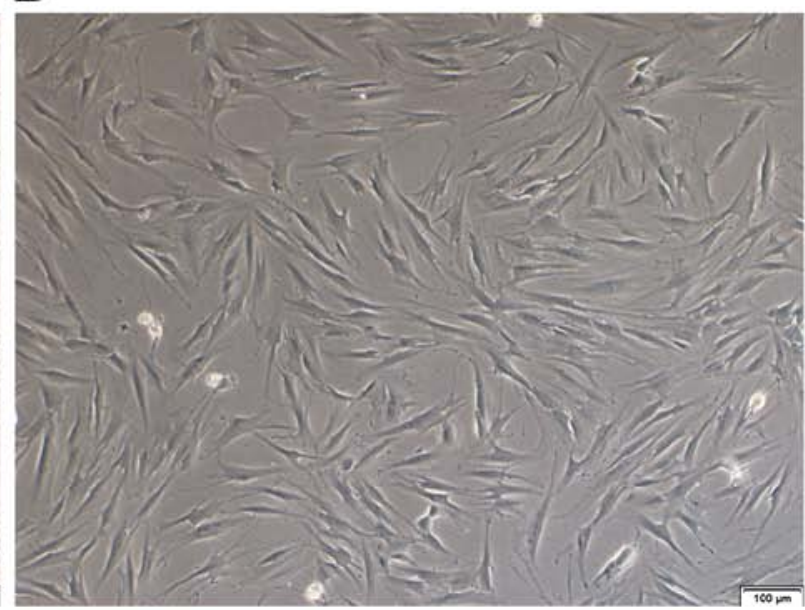

Figure 2. Differential characteristics of CAFs and PTFs in terms of morphology and growth manner. CAFs and PTFs were long spindle shaped. (A) CAFs were variable in size, with few and small cytoplasmic protrusions; growing in an overlapping manner. (B) PTFs performed more cytoplasmic processes, invariable in cell size, and could be characterized by contact and density inhibition. CAFs, cancer-associated fibroblasts; PTFs, peri-tumor fibroblasts.

$\mathrm{KGaA}$ ) according to the manufacturer's protocol. Quantitative analyses were performed using ImageJ software (version 1.46; National Institutes of Health, Bethesda). GAPDH (dilution, 1:2,000; cat. no. 60004-1-Ig; ProteinTech Group, Inc.) was used as an internal control.

Cell viability analysis. Purified CAFs and PTFs (4x10 $0^{3}$ cells/well) were loaded onto 96 -well plates in $100 \mu \mathrm{l}$ complete medium (DMEM containing $1 \%$ penicillin-streptomycin and $10 \% \mathrm{FBS}$ ) and maintained in $\mathrm{a} \mathrm{CO}_{2}$ incubator at $37^{\circ} \mathrm{C}$ for $24 \mathrm{~h}$. Each group contained five parallel wells, as well as a negative control (without cells). Cells were monitored for 7 consecutive days using a Cell Counting Kit-8 (CCK-8) kit (Dojindo Molecular Technologies, Inc.) following the manufacturer's instructions. The CCK-8 agent (10 $\mu \mathrm{l})$ was added to each well and incubated for $1 \mathrm{~h}$ at $37^{\circ} \mathrm{C}$ in the dark. Absorbance values [optical density (OD)] values at $450 \mathrm{~nm}$ were measured using a microplate reader.
Reverse transcription-quantitative polymerase chain reaction $(R T-q P C R)$. CAFs and PTFs were seeded into 6-well plates $\left(1.5 \times 10^{5}\right.$ cells/well) and cultured in DMEM supplemented with $10 \%$ FBS for $24 \mathrm{~h}$. Total RNA from these fibroblasts was isolated using TRIzol reagent (Invitrogen; Thermo Fisher Scientific), and $1 \mu \mathrm{g}$ of the extracted RNA was used to synthesize cDNA according to the manufacturer's recommendations for PrimeScript RT master mix (Takara Biotechnology Co., Ltd.). The mRNA levels of secreted matrix metalloproteinase 2 (MMP2), stromal cell derived factor1 (SDF-1) and transforming growth factor $\beta 1$ (TGF $\beta 1$ ) were determined using a SYBR PremixTaq II kit (Takara Biotechnology Co., Ltd.) and a Roche 480 Light Cycler. GAPDH was used as a reference gene. The oligonucleotide sequences of the qPCR primers were as follows: GAPDH: Forward 5'-GCACCGTCAAGGCTG AGAAC-3' and reverse 5'-TGGTGAAGACGCCAGTGGA-3'; MMP2: Forward 5'-CCTGCAAGTTTCCATTCCGC-3' and reverse 5'-AACAGTGGACATGGCGGTC-3'; SDF-1: Forward 

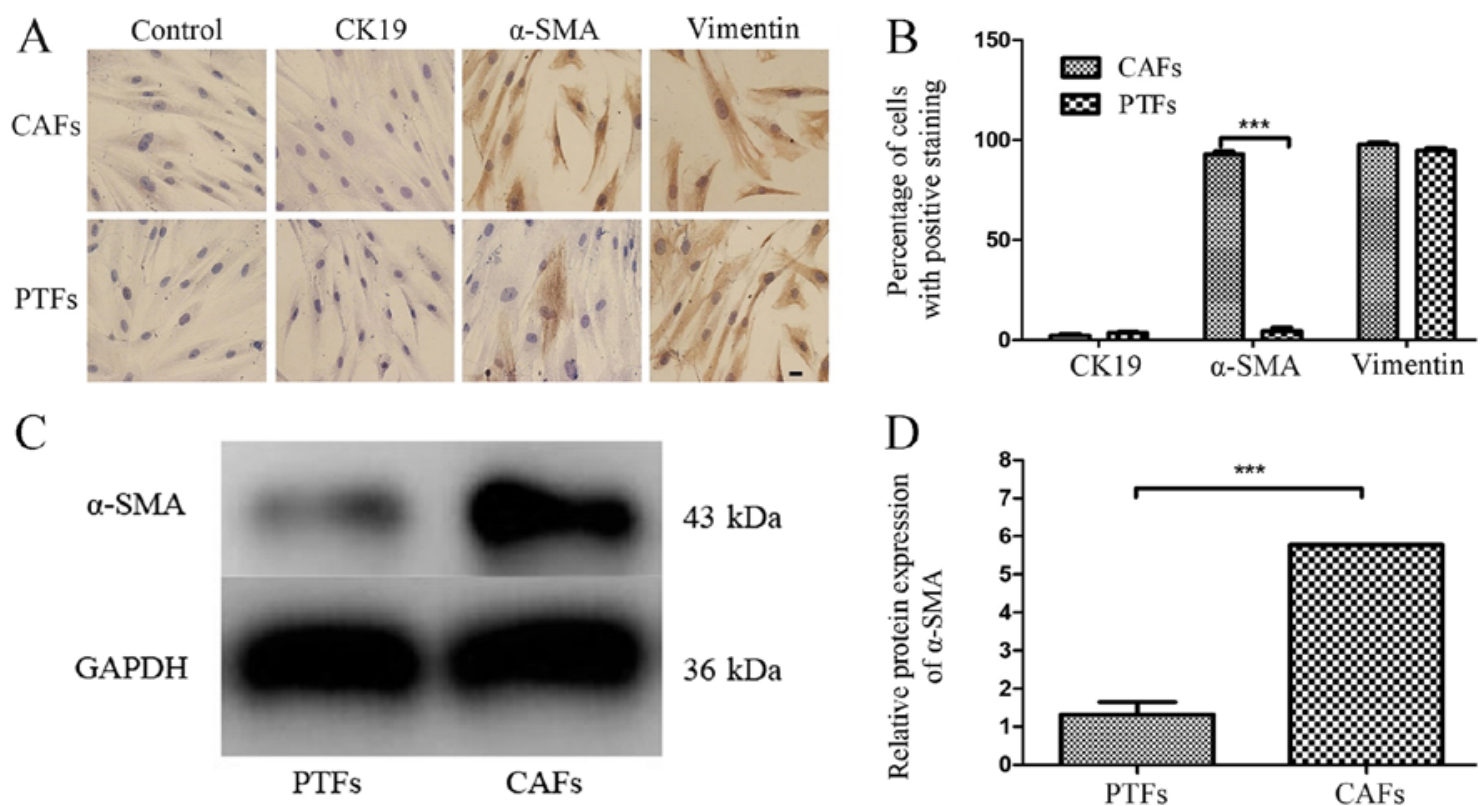

Figure 3. Immunophenotypic differences in CAFs and PTFs. (A) IHC staining and (B) analysis results showed that the percentage of $\alpha$-SMA positive cells in CAFs was significantly higher than that in PTFs $(\mathrm{P}<0.001)$, whereas there was no significant difference in the percentages of CK19- and vimentin-positive cells between CAFs and PTFs (P>0.05). (C) Western blot analysis and (D) quantitative analysis further confirmed that the expression of $\alpha$-SMA in CAFs was significantly higher compared with that in PTFs. The scale bar is equal to $20 \mu \mathrm{m},{ }^{* * *} \mathrm{P}<0.001$. IHC, immunohistochemistry; CAFs, cancer-associated fibroblasts; PTFs, peri-tumor fibroblasts; $\alpha$-SMA; $\alpha$-smooth muscle actin; CK19, cytokeratin 19.

5'-ATTCTCAACACTCCAAACTGTGC-3' and reverse 5'-ACT TTAGCTTCGGGTCAATGC-3; and TGF $\beta 1$ : Forward 5'-CGA CTCGCCAGAGTGGTTAT-3' and reverse 5'-CGGTAGTGA ACCCGTTGATGT-3'. Relative mRNA changes of the target genes were calculated using the $2^{-\Delta \Delta \mathrm{Cq}}$ method (17).

ELISA. CAFs and PTFs were cultured in 6-well plates $\left(1.5 \times 10^{5}\right.$ cells/well) with serum-free DMEM for $24 \mathrm{~h}$, after which the conditioned medium (CM) was harvested for ELISA analysis using the method described by Wang et al (18). The concentrations of MMP2, SDF-1 and TGF $\beta 1$ in CAFs- or PTFs-derived CM were determined using ELISA kits (cat. no. 70-EK1M022, 70-EK11192 and 70-EK1812, respectively; Multisciences Biotech Co., Ltd.) according to the manufacturer's instructions.

Statistical analysis. Statistical analysis was performed using SPSS 21.0 software (IBM Corp.). Values were compared using the Student's t-test. Data are presented as the means \pm standard deviation $\mathrm{P}<0.05$ was considered to indicate a statistically significant difference.

\section{Results}

Separation, cultivation and purification of CAFs and PTFs. The epidermoid cells and/or fibroblasts grew from the edge of the tissue. The epithelioid cells grew in clusters and exhibited dominant growth in the first few days, while the fibroblasts (CAFs or PTFs) grew radially along the tissue block or surrounding the clusters of epidermoid cells. The time taken for CAFs to migrate out of the tissue block is usually 4-6 days, faster than for PTFs (5-7 days after inoculation). It took 10-12 days for CAFs and 12-14 days for PTFs to reach 90\%

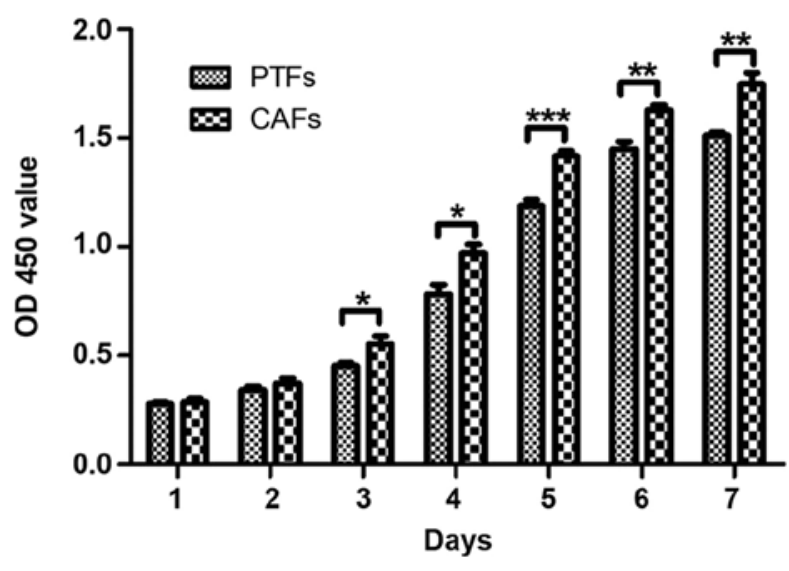

Figure 4. CAFs and PTFs viability assay. The OD450 value of CAFs was higher compared with that in PTFs from the third day of inoculation until the seventh day. Therefore, the viability of CAFs is higher compared with that of PTFs. ${ }^{*} \mathrm{P}<0.05,{ }^{* *} \mathrm{P}<0.01,{ }^{* * * *} \mathrm{P}<0.001$. OD, optical density; CAFs, cancer-associated fibroblasts; PTFs, peri-tumor fibroblasts.

confluence in a Petri dish. In P0-P2 cells, the fibroblasts mixed with the epithelioid cells. A mechanical curettage method (13) combined with trypsinization was used to purify these fibroblasts for $\mathrm{P} 0-\mathrm{P} 2$, and no epithelioid cells or tumor cells were mixed in with the cells after the P3 generation (Fig. 1).

Different morphology of CAFs and PTFs. There were differences in the morphology and growth mode between CAFs and PTFs. Morphological observation of CAFs and PTFs under an inverted microscope showed that the fibroblasts had a long spindle shape. CAFs usually have small cytoplasmic protrusions, which are accumulated and overlap in a disorderly arrangement, and presented visible loss of contact inhibition, 

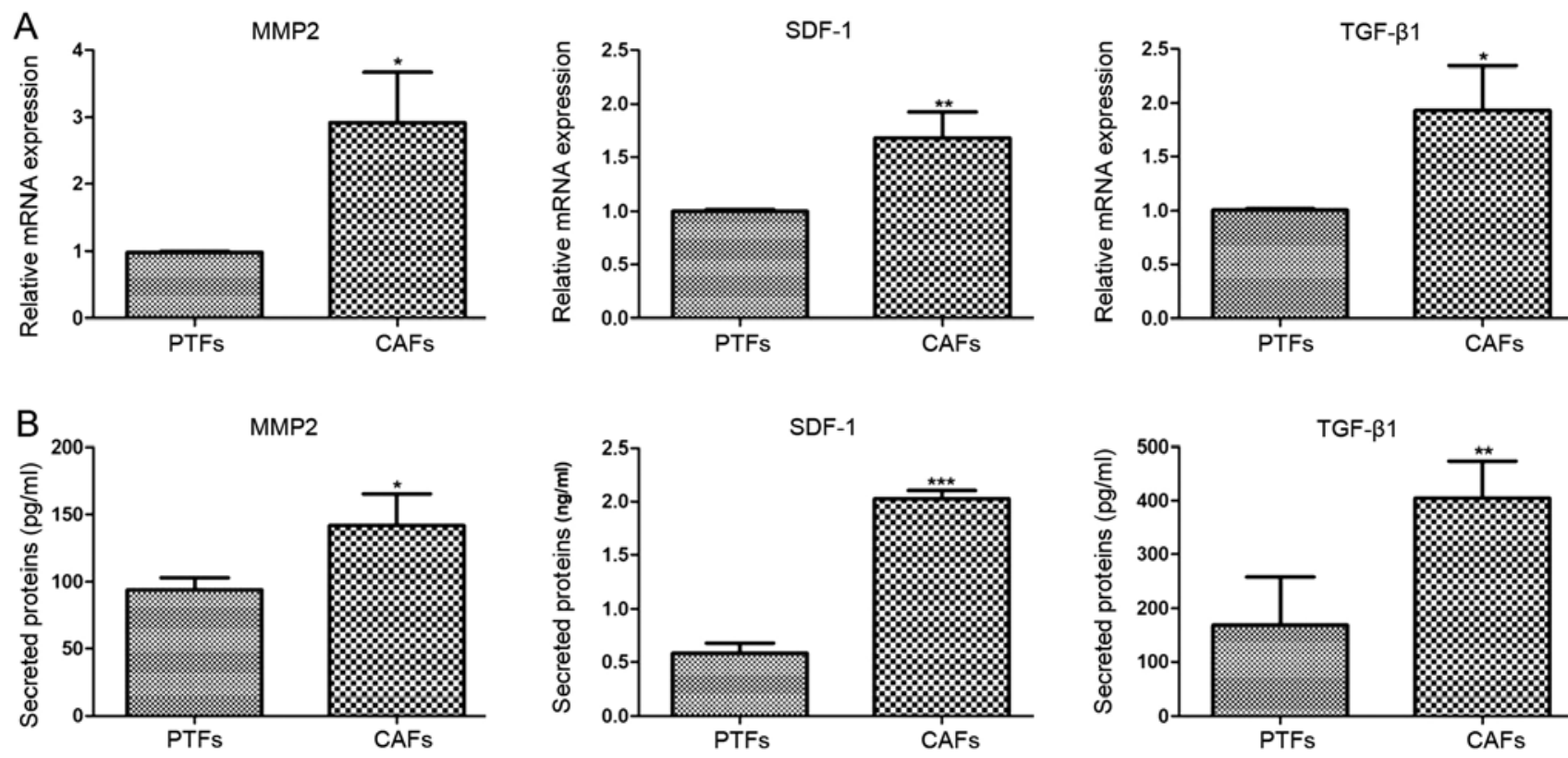

Figure 5. Differential expression of pro-carcinogenic cytokine in CAFs and PTFs. (A) mRNA expression analysis. CAFs expressed higher levels of MMP2, SDF-1 and TGF $\beta 1$ mRNA relative to that in PTFs. (B) ELISA results. CAFs secreted higher protein levels of MMP2, SDF-1 and TGF $\beta 1$ compared with that that in PTFs. ${ }^{*} \mathrm{P}<0.05,{ }^{* *} \mathrm{P}<0.01,{ }^{* * *} \mathrm{P}<0.001$. CAFs, cancer-associated fibroblasts; PTFs, peri-tumor fibroblasts; MMP2, matrix metalloproteinase 2; SDF-1; stromal cell derived factorl; TGF $\beta 1$, transforming growth factor $\beta 1$.

which is characteristic of the proliferation of malignant cells (19). By contrast, PTFs seem to have more cytoplasmic processes and were nearly invariable with respect to size; the cells showed obvious contact and density inhibition (Fig. 2).

Different immunophenotypical characterization of PTFs and CAFs. To observe the immunophenotype of PTFs and CAFs, the expression of vimentin, $\alpha$-SMA (markers for mesenchymal cells) and CK19 (marker for epithelial cells) were detected in these fibroblasts using IHC staining. The IHC results showed that both CAFs and PTFs had positive expression of vimentin and negative expression of CK19. CAFs positively expressed $\alpha$-SMA, while PTFs showed negative or only partly positive expression for $\alpha$-SMA (Fig. 3A). The percentages of CAFs and PTFs with positive expression of CK19, $\alpha$-SMA or vimentin were calculated (Fig. 3B). The results show that the percentage of $\alpha$-SMA-positive cells in CAFs was significantly higher compared with that in PTFs $(92.8 \pm 2.05$ vs. $4.4 \pm 2.5 \%$; $\mathrm{P}<0.001)$. However, there was no significant difference in the percentage of positive staining for CK19 (2.4 41.0 vs. $3.5 \pm 0.6 \%$; $\mathrm{P}>0.05)$ and vimentin $(97.7 \pm 1.5$ vs. $94.5 \pm 2.2 \% ; \mathrm{P}>0.05)$ in CAFs compared with that in PTFs (Fig. 3B). The expression of $\alpha$-SMA in CAFs and PTFs was further confirmed using western blot analysis for quantitative analysis, and the results showed that the expression of $\alpha$-SMA in CAFs was 4 -fold higher compared with that in PTFs $(\mathrm{P}<0.001$; Fig. $3 \mathrm{C}$ and $\mathrm{D})$. These data demonstrate that $\alpha$-SMA may serve as an excellent marker for distinguishing CAFs from PTFs.

Different viability of CAFs and PTFs. A CCK-8 kit was used to detect the viability of CAFs and PTFs. To ensure the same culture conditions, equal quantities of the cells $\left(4 \times 10^{3}\right)$ were inoculated in 96 well plates, and the time of CCK- 8 incubation and detection was the same every day. From day 3-7 after inoculation, the OD450 value of CAFs increased compared with that of PTFs. These results showed that the viability of CAFs was significantly higher compared with that of PTFs $(\mathrm{P}<0.05, \mathrm{P}<0.01$ and $\mathrm{P}<0.001$; Fig. 4).

Differential expression of pro-carcinogenic cytokines in CAFs and PTFs. CAFs and PTFs were cultured in DMEM containing $10 \%$ FBS for $24 \mathrm{~h}$. To detect the mRNA expression of pro-carcinogenic cytokines (MMP2, SDF-1 and TGF 11 ) RT-qPCR was performed. The expression of MMP2, SDF-1 and TGF $\beta 1$ in CAFs were 2.97-, 1.68- and 1.92-fold higher compared with that in PTFs $(\mathrm{P}<0.05, \mathrm{P}<0.01, \mathrm{P}<0.05$, respectively; Fig. 5A). Since these pro-carcinogenic cytokines are secretory proteins, the levels of solubilized MMP2, SDF-1 and TGF $\beta 1$ protein in the CM of CAFs and PTFs were assessed using corresponding ELISA kits. The concentrations of MMP2, SDF-1 and TGF 31 in CAFs were 1.51-, 3.47- and 2.40-fold higher compared with those in PTFs. Thus, it was confirmed that CAFs expressed significantly higher mRNA and protein levels of MMP2, SDF-1 and TGF $\beta 1$ compared with PTFs $(\mathrm{P}<0.05, \mathrm{P}<0.001$ and $\mathrm{P}<0.01$, respectively; Fig. 5B).

\section{Discussion}

There are several differences between CAFs and NFs; in particular, cell morphology, proliferation, secretory function and the expression of certain surface markers (such as $\alpha$-SMA; fibroblast activation protein, FAP; fibroblast specific protein 1, FSP1) $(20,21)$. However, whether these features distinguish CAFs from PTFs in TSCC is still unclear. During carcinogenesis, the changes occurring within cells are not only restricted to epithelial cells, but also occur in stromal cells. Following changes in epithelial cancer cells, various cells, such as resident fibroblasts, adipocytes, epithelial cells, endothelial cells or bone 
marrow derived mesenchymal stem cells, were phenotypically and functionally modified and differentiated into CAFs in the TME $(22,23)$. Cancer cells play an important role in inducing the transformation of local fibroblasts into CAFs, and in endowing CAFs with pro-carcinogenic effects by secreting cytokines such as TGF $\beta$ and IL-1 $\beta$ (24), thus leading to differences between CAFs and PTFs in terms of cell morphology, viability, secretory function and surface marker expression.

To study the changes to stromal cells during tumor progression, the purified fibroblast populations were verified according to cell morphology under a microscope, and using immunostaining, cell viability analysis and pro-carcinogenic cytokine expression. Observation under a phase-contrast microscope showed that, compared with CAFs, PTFs performed more cytoplasmic processes, were invariable in size and showed contact and density inhibition.

Previous studies have reported that CAFs express vimentin (mesenchymal marker) and $\alpha$-SMA (a specific marker of active fibroblasts), but do not express cytokeratin (maker of epithelial cells) $(13,14,25)$, though they are usually derived from various origins. Therefore, the expression of vimentin, $\alpha$-SMA and cytokeratin was detected to identify the immunophenotype of the primarily cultured fibroblasts. $\alpha$-SMA is not only a widely used marker for identifying CAFs, but is also an independent prognostic marker, which significantly correlated with metastasis, recurrence and mortality in oral squamous cell carcinoma (OSCC) (26-28). It has been reported that the expression of $\alpha$-SMA in PTFs is variable in different organs $(29,30)$. In the present study, the expression of $\alpha$-SMA in purified CAFs was significantly higher compared with that in PTFs in TSCC detected by IHC. Western blot analysis further verified that CAFs expressed high levels of $\alpha$-SMA relative to that in PTFs. These results confirmed that the expression of $\alpha$-SMA is increased step by step in PTFs and CAFs, which indicated that the fibroblasts may perform partial immunophenotypic changes earlier than the epithelial cells.

A study on the morphological, functional and genetic alteration of NFs, PTFs and CAFs in normal tissue, peri-tumor tissue and tumoral tissue would be beneficial to further reveal the processes involved in tumor progression. In general, peri-tumor tissue described in the literature refers to the tissues with no abnormal histological appearance at a certain distance around the tumor boundary. In a previous study, fibroblasts derived from normal oral mucosa or para-cancerous tissue at least $2 \mathrm{~cm}$ away from the outer tumor margin were more commonly used as control cells (31). In the present study, fibroblasts derived from peri-tumor tissues $1 \mathrm{~cm}$ away from the outer tumor margin were not only significantly different from the biological characteristics of CAFs, but also retained the morphology and function of the tongue tissue to a greater extent.

Paracrine signaling is an important way for tumor stromal cells to participate in tumorigenesis and development. In the present study, CAFs showed significant pro-carcinogenic potential compared with that in PTFs. It has recently become widely accepted that CAFs could be an efficient target for cancer therapy (32). Drugs that can regulate the phenotype and function of CAFs cells may be used in the treatment of tumors. In future studies, it is necessary to further explore the differential performance in chemosensitivity between CAFs and PTFs, which will be beneficial to the therapy of TSCC.
In conclusion, PTFs are distinguishable from CAFs in terms of their biological characteristics, and PTFs would be suitable control cells in a study investigating the role of CAFs in tumorigenesis and tumor progression.

\section{Acknowledgements}

Not applicable.

\section{Funding}

This investigation was supported by Natural Science Foundation of China (grant no. 81702684), Beijing, China, and Shandong Medical and Health Science and Technology Development Plan Project, Jinan, Shandong (grant no. 2018WS112).

\section{Availability of data and materials}

The datasets used and/or analyzed during the present study are available from the corresponding author on reasonable request.

\section{Authors' contributions}

$\mathrm{PB}$ and $\mathrm{XZ}$ performed the experiment and wrote the manuscript. QS and CY were responsible for the design of the experiment. MY, LL, GF and PY analyzed the experimental data and assisted in the revision of the manuscript. XD, MW, and SL assisted with the statistical analysis. All authors read and approved the final manuscript.

\section{Ethics approval and consent to participate}

All research procedures were approved by the Medical Ethics Committee of Qilu Hospital, Shandong University (approval no. 2016015) and informed consent was obtained from all the participants.

\section{Patient consent for publication}

Not applicable.

\section{Competing interests}

The authors declare that they have no competing interests.

\section{References}

1. Torre LA, Bray F, Siegel RL, Ferlay J, Lortet-Tieulent J and Jemal A: Global cancer statistics, 2012. CA Cancer J Clin 65: 87-108, 2015.

2. Qiu K, Huang Z, Huang Z, He Z and You S: miR-22 regulates cell invasion, migration and proliferation in vitro through inhibiting CD147 expression in tongue squamous cell carcinoma. Arch Oral Biol 66: 92-97, 2016.

3. Tsushima N, Sakashita T, Homma A, Hatakeyama H, Kano S, Mizumachi T, Kakizaki T, Suzuki T and Fukuda S: The role of prophylactic neck dissection and tumor thickness evaluation for patients with cN0 tongue squamous cell carcinoma. Eur Arch Otorhinolaryngol 273: 3987-3992, 2016.

4. Zhang T, Liang L, Liu X, Wu JN, Chen J, Su K, Zheng Q, Huang $\mathrm{H}$ and Liao GQ: TGF $\beta 1$-Smad3-Jagged1-Notch1-Slug signaling pathway takes part in tumorigenesis and progress of tongue squamous cell carcinoma. J Oral Pathol Med 45: 486-493, 2016. 
5. Rodrigues-Lisoni FC, Peitl P Jr, Vidotto A, Polachini GM, Maniglia JV, Carmona-Raphe J, Cunha BR, Henrique T, Souza CF, Teixeira RA, et al: Genomics and proteomics approaches to the study of cancer-stroma interactions. BMC Med Genomics 3: 14, 2010.

6. Kalluri R and Zeisberg M: Fibroblasts in cancer. Nat Rev Cancer 6: 392-401, 2006.

7. Jia CC, Wang TT, Liu W, Fu BS, Hua X, Wang GY, Li TJ, Li X, Wu XY, Tai Y, et al: Cancer-associated fibroblasts from hepatocellular carcinoma promote malignant cell proliferation by HGF secretion. PLoS One 8: e63243, 2013.

8. Du H and Che G: Genetic alterations and epigenetic alterations of cancer-associated fibroblasts. Oncol Lett 13: 3-12, 2017.

9. Xouri G and Christian S: Origin and function of tumor stroma fibroblasts. Semin Cell Dev Biol 21: 40-46, 2010.

10. Karagiannis GS, Poutahidis T, Erdman SE, Kirsch R, Riddell RH and Diamandis EP: Cancer-associated fibroblasts drive the progression of metastasis through both paracrine and mechanical pressure on cancer tissue. Mol Cancer Res 10: 1403-1418, 2012

11. Beacham DA and Cukierman E: Stromagenesis: The changing face of fibroblastic microenvironments during tumor progression. Semin Cancer Biol 15: 329-341, 2005.

12. Xing F, Saidou J and Watabe K: Cancer associated fibroblasts (CAFs) in tumor microenvironment. Front Biosci (Landmark Ed) 15: 166-179, 2011.

13. Liu Y, Hu T, Shen J, Li SF, Lin JW, Zheng XH, Gao QH and Zhou HM: Separation, cultivation and biological characteristics of oral carcinoma-associated fibroblasts. Oral Dis 12: 375-380, 2006.

14. Zhou B, Chen WL, Wang YY, Lin ZY, Zhang DM, Fan S and Li JS: A role for cancer-associated fibroblasts in inducing the epithelial-to-mesenchymal transition in human tongue squamous cell carcinoma. J Oral Pathol Med 43: 585-592, 2014.

15. Brezinski D, Stone PH, Muller JE, Tofler GH, Davis V, Parker C, Hartley LH and Braunwald E: Prognostic significance of the Karnofsky Performance Status score in patients with acute myocardial infarction: Comparison with the left ventricular ejection fraction and the exercise treadmill test performance. The MILIS Study Group. Am Heart J 121: 1374-1381, 1991.

16. Sobin LH, and Wittekind C: TNM classification of malignant tumours. 7th edition, Wiley-Blackwell, Hoboken, NJ, pp309, 2010.

17. Livak KJ and Schmittgen TD: Analysis of relative gene expression data using real-time quantitative PCR and the 2(-Delta Delta C(T)) method. Methods 25: 402-408, 2001.

18. Wang L, Zhang F, Cui JY, Chen L, Chen YT and Liu BW: CAFs enhance paclitaxel resistance by inducing EMT through the IL6/JAK2/STAT3 pathway. Oncol Rep 39: 2081-2090, 2018.

19. Hanahan D and Weinberg RA: Hallmarks of cancer: The next generation. Cell 144: 646-674, 2011.

20. Wang L, Cao L, Wang H, Liu B, Zhang Q, Meng Z, Wu X, Zhou Q and $\mathrm{Xu} \mathrm{K}$ : Cancer-associated fibroblasts enhance metastatic potential of lung cancer cells through IL-6/STAT3 signaling pathway. Oncotarget 8: 76116-76128, 2017.
21. Räsänen K, Virtanen I, Salmenperä P, Grenman R and Vaheri A Differences in the nemosis response of normal and cancer-associated fibroblasts from patients with oral squamous cell carcinoma. PLoS One 4: e6879, 2009.

22. Kuzet SE and Gaggioli C: Fibroblast activation in cancer: When seed fertilizes soil. Cell Tissue Res 365: 607-619, 2016.

23. Shiga K,Hara M,Nagasaki T, Sato T, Takahashi H and Takeyama H: Cancer-associated fibroblasts: Their characteristics and their roles in tumor growth. Cancers (Basel) 7: 2443-2458, 2015.

24. Leef G and Thomas SM: Molecular communication between tumor-associated fibroblasts and head and neck squamous cell carcinoma. Oral Oncol 49: 381-386, 2013.

25. Liu J, Chen S, Wang W, Ning BF, Chen F, Shen W, Ding J, Chen W, Xie WF and Zhang X: Cancer-associated fibroblasts promote hepatocellular carcinoma metastasis through chemokine-activated hedgehog and TGF- $\beta$ pathways. Cancer Lett 379: 49-59, 2016.

26. Dourado RC, Porto LPA, Leitão ÁCGH, Cerqueira PSG, Dos Santos JN, Ramalho LMP and Xavier FCA: Immunohistochemical characterization of Cancer-associated fibroblasts in oral squamous cell carcinoma. Appl Immunohistochem Mol Morphol 26: 640-647, 2018.

27. Marsh D, Suchak K, Moutasim KA, Vallath S, Hopper C, Jerjes W, Upile T, Kalavrezos N, Violette SM, Weinreb PH, et al: Stromal features are predictive of disease mortality in oral cancer patients. J Pathol 223: 470-481, 2011.

28. Luksic I, Suton P, Manojlovic S, Virag M, Petrovecki M and Macan D: Significance of myofibroblast appearance in squamous cell carcinoma of the oral cavity on the occurrence of occult regional metastases, distant metastases, and survival. Int J Oral Maxillofac Surg 44: 1075-1080, 2015.

29. Mazzocca A, Dituri F, Lupo L, Quaranta M, Antonaci S and Giannelli G: Tumor-secreted lysophostatidic acid accelerates hepatocellular carcinoma progression by promoting differentiation of peritumoral fibroblasts in myofibroblasts. Hepatology 54: 920-930, 2011

30. Hawsawi NM, Ghebeh H, Hendrayani SF, Tulbah A, Al-Eid M, Al-Tweigeri T, Ajarim D, Alaiya A, Dermime S and Aboussekhra A: Breast carcinoma-associated fibroblasts and their counterparts display neoplastic-specific changes. Cancer Res 68: 2717-2725, 2008.

31. Zhou B, Zhuang XM, Wang YY, Lin ZY, Zhang DM, Fan S, Li JS and Chen WL: Tumor necrosis factor $\alpha$ induces myofibroblast differentiation in human tongue cancer and promotes invasiveness and angiogenesis via secretion of stromal cell-derived factor-1. Oral Oncol 51: 1095-1102, 2015.

32. Alkasalias T, Moyano-Galceran L, Arsenian-Henriksson M and Lehti K: Fibroblasts in the tumor microenvironment: Shield or Spear? Int J Mol Sci 19: pii: E1532, 2018.

This work is licensed under a Creative Commons Attribution-NonCommercial-NoDerivatives 4.0 International (CC BY-NC-ND 4.0) License. 